\title{
RP-UHPLC-DAD-QTOF-MS as a powerful tool of oleuropein and ligstroside characterization in olive-leaf extract and their contribution to the improved performance of refined olive-pomace oil during heating
}

Ibtissem Ben Hammouda, Gloria Márquez-Ruiz, Francisca Holgado, Ammar Sonda, Krystyna Skalicka-Wozniak, and Mohamed Bouaziz*

\begin{abstract}
Since olive leaf is a potential source of phenolic fraction that is assumed to have good antioxidative effects, we purposed to add its extract to the refined olive-pomace oil during heating to increase its oxidative stability. RP-UHPLC-DAD-QTOF-MS was employed to characterize the phenolic fraction. The oil samples were evaluated by measuring the polymers and the polar compounds and thus detecting specific oxidized compounds. Using this approach, the results showed that incorporating olive leaf extract in refined oil significantly reduced the formation of polymers from $14.39 \%$ to $10.45 \%$ and the oxidation state by the variation of extinction $\Delta K$ from 3.02 to 2.29 during $20 \mathrm{~h}$ of heating compared to unenriched oil. This study has proven that the use of natural substances is an opportunity to extend the life of refined oils.
\end{abstract}

KEYWORDS: olive leaf extract, refined oils, rancimat, total polar compounds, phenolic compounds, RP-UHPLC-DAD-QTOF-MS 


\section{INTRODUCTION}

Among the method of food preparation, frying represents the most common one domestically and commercially. This process which is known as one of the oldest methods of cooking ${ }^{1}$ leads to exceptional sensory characteristics of color, flavor, texture, and palatability, extremely preferred by consumers.

Deep frying carries challenges to oil quality due to its exposition at elevated temperatures in the presence of moisture and oxygen. ${ }^{2}$ Hence, it is necessary to choose the most suitable oil for frying.

Various complex chemical reactions, namely hydrolysis, oxidation, cyclization, isomerization, and polymerization, occurring during deep-frying affect the quality features of the frying oil and ultimately the product properties such as texture, flavor, shelf life, and nutritious composition. ${ }^{2}$ It's worth noting that each vegetable oil is distinguished by a emblematic stability against oxidation, contingent upon the composition of the fatty acid and the content of minor compounds. Hence, an urgent need has emerged to delineate the ultimate changing point of the fat or oil, which is mainly associated with the content of total polar compounds (TPC) and the designed polymers that offer paramount techniques acclaimed for assessing frying dilapidation. Furthermore, the majority of European countries have set up regulations limiting the decay of the frying fats and oils employed for human consumption, with a maximally accepted content of polar compounds calculated at $25 \%$ or $12 \%$ polymers. ${ }^{3}$ Therefore, the choice of new industrial frying oils should meet several requirements.

Good flavor and oxidative stability are among the criteria that should be found in a vegetable oil utilized for frying to attain superior mean life for the fried product. Refined olive pomace oil has been used in preparing an ultimate mixture of frying oil. Similar to most of vegetable oils, it comprises various minor components like the tocopherols which are advantageous to oil stability. ${ }^{4}$

At elevated temperatures, oxidative stability is one of the most important features frying oil must have.

Although an oil with an elevated saturated fatty acid content is very heat-stable, it has adverse effects on health. An oil having important polyunsaturated fatty acids (PUFA) levels, which are recognized for their considerable health advantages, is inapproriate for 
frying because of the greater susceptibility to oxidation. Actually, to delay lipid oxidation in oils and fats, some techniques have been used. Thus, antioxidant treatment of the oil is likely to be a solution to the oxidation and instability problems of vegetable oils together with the enhancement of their oxidative stability. This treatment can occur throughout its use. However, many studies have been carried out on synthetic antioxidants such as butylatedhydroxytoluene (BHT) and tert-butylhydroquinone (TBHQ) that were added to frying oils to prevent their oxidation. These antioxidants have been shown to be highly effective during the storage and transportation of oils. ${ }^{5}$ Nonetheless, they are less operational at frying temperatures due to their volatility. Therefore, they have a harmful effect on health, which limits their use. Nevertheless, the presence of natural antioxidants such as tocopherols have demonstrated a great improvement in the performance of the frying oil at higher temperatures. ${ }^{6}$ Recently, to ameliorate the stability of oils and delay their oxidations, the addition of extracts of natural antioxidants in dietary lipids, such as olive leaf extract, has become a preferable practice. ${ }^{7}$ Olive leaves are one of the byproducts that may be obtained with great quantity in olive oil industries. They represent a low-priced raw material which may be employed as a prized source of high value-added products. Consequently, olive leaves have gained the interest of several researchers, scientists, and manufacturers all over the world, thanks to the discovery of health promoting benefits by a great deal of scientific data. Their chemical constituents have been characterized, revealing that polyphenols show up as prevalent micronutrients. ${ }^{8}$

Nowadays, bioactive compounds from olive leaves are likely to draw the attention of pharmaceutical, cosmetic, and food industries due to their great potential as antioxidant, hypolipidemic, anticarcinogenic, anti-inflammatory, and antihypertensive agents.

Indeed, many research works have highligted the health advantages of the extracts of olive leaf from their antioxidant characteristics through the bioavailability of certain phenolic compounds as well as their derivatives as tyrosol, oleuropein, ligstroside, hydroxytyrosol, ${ }^{9}$ etc. Likewise, the phenolic extracts of the olive plant (fruit, leaves, and pomace) have been evidenced to possess considerable antioxidant activity in delaying sunflower oil oxidative rancidity. ${ }^{5}$

Thus, this research work aims to demonstrate the effect of the addition of $1000 \mathrm{ppm}$ of olive-leaf extract rich in oleuropein and ligstroside on the quality and oxidative stability of refined olive-pomace oil before and after $20 \mathrm{~h}$ of heating. 


\section{MATERIALS AND METHODS}

Oil Samples. The oil examined during the heating process was refined olive-pomace oil (ROPO), which was obtained from the National Oil Office of Sfax, Tunisia. ROPO was prepared in a quantity of $8 \mathrm{~g}$ of enriched and nonenriched oils with the extract of olive leaves. The samples were heated in the Rancimat apparatus at $180^{\circ} \mathrm{C}$ for $20 \mathrm{~h}$, and then they were taken over $2.5,5,7,10,12.5,15$, and $20 \mathrm{~h}$ of heating. These oil samples (each sample weights $=500 \mathrm{mg}$ ) were subsequently stored in dark glass flasks and stored at $-20^{\circ} \mathrm{C}$ until future analysis.

Plant Material. The experiments were performed on fresh leaves (Olea europaea L.; $200 \mathrm{~g}$ ) of the Chemlali variety, generally grown in Tunisia. In fact, the drying of leaves was performed on site in a Gold star microwave oven, thrice for $3 \mathrm{~min}$ at maximum power $(1250 \mathrm{~W})$.

After being dried, the leaves were finely ground using an electric grinder (Moulinex 1.5-L Blender and Herbs Grinder (LM1A0D10)- France) to facilitate the extraction of all of the phenolic compounds and subsequently stored in closed dark glass flasks and stored at room temperature $-20^{\circ} \mathrm{C}$.

Chemicals and Standards. For extraction, we used ultrapure water and ethanol as solvents. All solvents utilized for LC-MS analysis were purchased from J.T Baker (Phillipsburg, U.S.A.). The reagent applied to assess the antioxidant capacity was FolinCiocalteau which was acquired from Fluka Chemika (Buchs, Switzerland).

Extraction of Phenolic Compounds from Olive Leaf. The selected method of extraction of our compounds ${ }^{5}$ was as follows. A total of $7 \mathrm{~g}$ of olive leaf powder obtained above was extracted with a blend of ethanol/water $(75 \mathrm{~mL}, 70: 30 \mathrm{v} / \mathrm{v})$. The set was homogenized at room temperature with a vortex for $1 \mathrm{~min}$.

Subsequently, the solution was stirred in a shaker at $12-15 \mathrm{rpm}$ overnight at dark and ambient temperature. The obtained solution was then filtered using GF/F filter paper. The concentration of the extract was realized under vacuum and with a rotary evaporator without exceeding $45^{\circ} \mathrm{C}$, and then, the resulting residue was stored in a sealed dark glass vial and stored at $0{ }^{\circ} \mathrm{C}$. Eventually, the resulting residue was dissolved in $25 \mathrm{~mL}$ of ethanol and a quantity of $1000 \mathrm{ppm}$ of the prepared extract was added to the frying oil. 
RP-UHPLC-DAD-QTOF-MS and -MS/MS Analysis. The analyses were carried out with on Agilent 1200 series equipped with a binary pump, an autosampler equipped and a DAD (Agilent Technologies, Palo Alto, CA, USA). The system was coupled with a 6540 Agilent Ultra-High-Definition Accurate-Mass, equipped with Agilent Dual Jet Stream electrospray ionization (Dual AJS ESI) interface. For phenolic compounds' characterization, the mobile phases consisted in the incorporation of solvent $\mathrm{A}(1 \%$ acetonitrile with $0.1 \%$ ammonium formate $(10 \mathrm{mM}))$ and solvent $\mathrm{B}(95 \%$ acetonitrile with $0.1 \%$ ammonium formate $(10 \mathrm{mM}))$. The linear gradient and the flow rate were applied according to previous studies. ${ }^{10,16}$ The separation were also realized as previously described by Talhaoui et al. ${ }^{17}$

DPPH Radical Scavenging Assay. The testing procedure was carried out based on a previous study. ${ }^{11}$ In brief, we added aliquots $(50 \mathrm{~mL})$ of different concentrations of the tested compound in methanol to $5 \mathrm{~mL}$ of a $0.004 \%$ methanol solution of DPPH $(1,1-$ diphenyl-2-picrylhydrazyl). The mixture was incubated for $30 \mathrm{~min}$ at RT, then its absorbance was measured at $517 \mathrm{~nm}$ with UV-visible spectrophotometer.

The calculation of the percent value of radical scavenging represented by (IC50, expressed in $\mathrm{mg} / \mathrm{mL}$ ) value was conducted using the following formula: \% radical scavenging $=[(A$ blank $-A$ sample $) / A$ blank $] \times 100$, where $A$ blank is the absorbance of the control reaction (containing all reagents except the test compound) and $A$ sample is the absorbance of the test compound. We used the synthetic antioxidants BHT and ascorbic acid as positive controls.

Determination of Total Polar Compounds. The measurement of the total polar compounds was conducted directly in the oil with a Testo 270 instrument (Oil Tester, Testo Inc., Germany) during the frying process.

These components were estimated based on detecting the oil's dielectric constant, with the results given in percentage (\%). The analysis of the samples was realized by immersing the Testo sensor in hot oil at frying temperature. The measurements were taken after $10 \mathrm{~s}$. The TPC percentages and temperature values were exposed on the instrument's screen. ${ }^{4}$

Quantitation of Triacylglycerol Polymers. Oil samples $(50 \mathrm{mg}$ ) were weighed and dissolved in $1 \mathrm{~mL}$ of tetrahydrofuran to be later analyzed by high-performance sizeexclusion chromatography, using a Rheodyne $7725 \mathrm{i}$ injector with a $10-\mu \mathrm{L}$ sample loop, 
a Knauer 120 HPLC pump (Berlin, Germany), and a Merck L-7490 refractive index detector (Darmstadt, Germany).

The components were divided into two columns: 100 and 500 Ultrastyragel $(25 \mathrm{~cm} \times$ $0.77 \mathrm{~cm}$ i.d.) crammed with porous, greatly cross-linked styrene-divinylbenzene copolymers (particle size $5 \mu \mathrm{m}$; Hewlett-Packard, Avondale, PA, U.S.A.) linked in series to tetrahydrofuran $(1 \mathrm{~mL} / \mathrm{min})$ as the mobile phase, following IUPAC Standard Method 2.508. ${ }^{12}$ Polar components were identified according to the methods described by previous studies. ${ }^{12,13}$

Coefficients of Specific Extinction at 232 and $270 \mathrm{~nm}$ (K232 and K270). Particular absorptivity at 232 and $270 \mathrm{~nm}\left(K_{232}\right.$ and $\left.K_{270}\right)$ were carried out consistent with the International Olive Oil Council standards.14 An amount of $0.05 \mathrm{~g}$ of oil was dissolved in $25 \mathrm{~mL}$ of cyclohexane to determine the extinction coefficient $\mathrm{K} 232$ and $0.25 \mathrm{~g}$ to determine the extinction coefficient $K_{270}$.

Specific extinction was calculated from a spectrophotometer (model 35, Beckman Instruments, Inc., Fullerton, CA), utilizing pure cyclohexane as a blank.

The colorimetrical measurement of the total phenols were conducted at $725 \mathrm{~nm}$ with the Folin-Ciocalteu reagent. ${ }^{15}$

\section{RESULTS AND DISCUSSION}

Identification of Phenolic Compounds in Olive Leaf Extract. In this research work, the phenolic composition of the olive leaf extract was qualitatively analyzed using RPUHPLCDAD- QTOF-MS and MS/MS, by electrospray in negative ionization mode. The latter was used because it offered the uppermost sensitivity to achieve highresolution mass spectra. Figure 1 displays the base peaks chromatograms (BPCs) of the olive leaf extract obtained with the optimized analytical conditions. Table 1 exposes a synopsis of the phenolic compounds determined in olive leaves and their retention time (RT), molecular formula, molecular monoisotopic mass, experimental $\mathrm{m} / \mathrm{z}$, UV data (maximums, in $\mathrm{nm}$ ), main MS/MS fragments, double bond equivalents (DBE), and the proposed assignment. The accurate determinations of mass in the negative ionization mode facilitated the detection of each molecular ion's molecular formula. Such data along with the fragmentation pattern investigation in the literature as well as databases have permitted the validation of the proposed chemical structures. Whenever possible, a comparison of the compounds with standards in terms of RT, UV, MS, and MS/ MS 
spectral features was conducted. Moreover, by using the UV-vis detection data, the objective was essentially to distinguish the chemical structures, thus according well with the results found in previous studies. ${ }^{10,16}$

Yet, the DBE (total number of combine drings and double bonds in the molecule) value is announced as an index of aromaticity or/and instauration for each nominated compound.

In turn, the QTOF mass analyzer gives precise measurements of the mass and isotopic conformity, allowing the target compound's molecular formula to be obtained.

Identification of Compounds 1 and 2. The HPLCDAD- TOF-MS was analyzed the olive-leaf extract, revealing the already-reported compounds in olive leaf (Table 1).

Peaks 1 and 2 (Figure 1; RT 27.01 and 28.17 min, respectively) represent the molecular masses of $\mathrm{m} / \mathrm{z} 539.1771$ and $\mathrm{m} / \mathrm{z} 539.1774$, respectively. They are identified as oleuropein (isomers a and b), one of the most plentiful phenolic compounds of olive fruit $^{16,17}$ according to the molecular formula $\left(\mathrm{C}_{25} \mathrm{H}_{32} \mathrm{O}_{13}\right)$ conveyed for their mass, UV $(242,282 \mathrm{~nm})$ and their MS/MS spectra which provide almost identical fragmentation mechanisms.

As predicted, oleuropein is among the main components of the phenolic fraction that has been detected in olive leaf extracts. Furthermore, it shows antioxidant properties thanks to its catechol fraction. ${ }^{18}$

The ESI-MS2 spectrum of the $[\mathrm{M}-\mathrm{H}]^{-}$ion for the isomer (a) at $m / z 539.1771$ revealed the major product ions at $\mathrm{m} / \mathrm{z} 377.1241,307.0822,275.0920$, and 223.0610 (Figure 2).

The ion at $\mathrm{m} / \mathrm{z}$ 377.1241, observed in the $\mathrm{MS}^{2}$ spectrum, matches to oleuropein aglycone $\left(\mathrm{C}_{16} \mathrm{H}_{26} \mathrm{O}_{10}\right)$, and it is made by the loss of glucosyl (162.0530 Da). Moreover, the ion $\left(m / z\right.$ 307.0822) is vindicated by the $\mathrm{C}_{4} \mathrm{H}_{6} \mathrm{O}$ fragment loss from the ion at $(\mathrm{m} / z$ 377.1241; $\left.\mathrm{C}_{19} \mathrm{H}_{21} \mathrm{O}_{8}\right)$ [M-H-glucoside- $\left.\mathrm{C}_{4} \mathrm{H}_{6} \mathrm{O}\right]$ - Furthermore, the ion at $(\mathrm{m} / z$ 275.0920; $\mathrm{C}_{15} \mathrm{H}_{15} \mathrm{O}_{5}$ ) might be the result of a retro-type rearrangement Diels-Alder ${ }^{16}$ and the fragment ion at $(\mathrm{m} / \mathrm{z} 223.0610)$ can emanate from a rearrangement of the Mclafferty type of the ester function (Figure 3).

Identification of Compound 3. The product ions of compound 3 generated in LCMS/MS chromatogram (BPC) presented molecular ions at (m/z 523.1823, base peak), and eluted at a retention time of 29.45 min (Figure 4) was assigned to Ligstroside. ${ }^{17,19}$ The chief product ions obtained in the $\mathrm{MS}^{2}$ spectrum of the $[\mathrm{M}-\mathrm{H}]^{-}$ion at $\mathrm{m} / z 523.1823$ were detected at $\mathrm{m} / \mathrm{z} 361.1291,291.0877$, and 259.0977 (Figure 5). 
In fact, the ion fragment at $m / z 361.1291\left(\mathrm{C}_{19} \mathrm{H}_{21} \mathrm{O}_{7}\right)$ obtained by the neutral loss of $162.0530 \mathrm{Da}\left(\mathrm{C}_{6} \mathrm{H}_{10} \mathrm{O}_{5}\right)$ [MHglucosyl] ${ }^{-}$can be explained by the elimination of the glucoside group which corresponds to ligstroside aglycone. ${ }^{19}$

The $\mathrm{MS}^{2}$ spectrum of the $[\mathrm{M}-\mathrm{H}]^{-}$ion at $\mathrm{m} / \mathrm{z} 523.1823$ exhibits product ions at $\mathrm{m} / \mathrm{z}$ 329.0899 and 291.0877 originating from the neutral loss of $\mathrm{CH}_{3} \mathrm{OH}$ group (32.0392 Da) and $\mathrm{C}_{4} \mathrm{H}_{6} \mathrm{O}$ group $(70.0414 \mathrm{Da})$ from the ion fragment at $\mathrm{m} / \mathrm{z} 361.1291$, which gave fragment ions $\left[\mathrm{MH}-\mathrm{CH}_{3} \mathrm{OH}\right]^{-}$and $\left[\mathrm{MH}-\mathrm{C}_{4} \mathrm{H}_{6} \mathrm{O}\right]^{-}$, respectively. They, in turn, formed the fragmentation ion $\left[\mathrm{MH}-\mathrm{C}_{4} \mathrm{H}_{6} \mathrm{O}-\mathrm{CH}_{3} \mathrm{OH}\right](\mathrm{m} / z$ 259.0977). The preliminary structure of these compounds is presented in Figure 6.

Besides, the ion at $m / z 139.0374\left(\mathrm{C}_{6} \mathrm{H}_{3} \mathrm{O}_{4}\right)$ was formed by the loss of $\mathrm{CH}_{2} \mathrm{CHPhOH}$ from the ligstroside aglycone ion at $\mathrm{m} / \mathrm{z} 361.1291$ and the loss of $\mathrm{C}_{8} \mathrm{H}_{8} \mathrm{O}$ (120.0603 Da; $\mathrm{m} / \mathrm{z} 259.0977$ ) by McLafferty rearrangement. Indeed, the observed ion at $\mathrm{m} / z 223.0610$ $\left(\mathrm{C}_{11} \mathrm{H}_{11} \mathrm{O}_{5}\right)$ can be explained by the loss of a molecule of water $\left(\mathrm{H}_{2} \mathrm{O}\right)$ after a McLafferty rearrangement (Figure 6).

Antiradical Effects of the Extract of Olive Leaf and Commercial Standards. The DPPH test identifies the antifree radical potency of antioxidants. The DPPH radical scavenging activity of the olive leaf extract and that of the commercial synthetic antioxidants as ascorbic acid $\left(\mathrm{IC}_{50}=5.00 \mu \mathrm{g} / \mathrm{mL}\right)$ and $\mathrm{BHT}\left(\mathrm{IC}_{50}=7.94 \mu \mathrm{g} / \mathrm{mL}\right)$ were measured and compared. The lower the $\mathrm{IC}_{50}$ values are the higher the free radical scavenging activity in the sample is. The extract has demonstrated that the substantial antioxidant activity is mostly efficient with an $\mathrm{IC}_{50}$ value of $2.34 \mu \mathrm{g} / \mathrm{mL}$ (Figure 7).

Influence of Frying on the Quality Characteristics of Refined Oils. Polar Compounds. The measurement of TPC and DPTG is reckoned to be the most exact evaluation of the cooking oils' oxidative degradation. Several countries have set up supervisory restrictions for TPC in cooking oils at 25\% and DPTG between 12 and $16 \%$ as the limit. ${ }^{1}$

To examine the impact of the extract of the olive leaf on the oxidative resistance of the refined oil samples before and after 2.5, 5, 7.5, 10, 12.5, 15, and $20 \mathrm{~h}$ of heating, the amounts of TPC and DPTG were determined. These amounts that are produced during frying are the major oil deterioration indicators. They are nonvolatile compounds that represent the main products of the reactions occurring in the heated oil ${ }^{20}$ due to thermal, hydrolytic, and oxidative alterations. 
Furthermore, the samples of enriched (ROPOE) and unenriched (ROPO) olive-pomace oils with the phenolic extract were heated at $180^{\circ} \mathrm{C}$ by the Rancimat method.

Indeed, the TPC content increased significantly from $8 \%$ to $11.5,16.5,23.5$, and $26 \%$ over $5,10,15$, and $20 \mathrm{~h}$ of heating, respectively, for unenriched oil (ROPO), whereas the enriched oil (ROPOE) showed a slow increase at the same order time from $8 \%$ to $8.3,11.5,17$, and $24.75 \%$, respectively.

Equally, for the sake of comparing the contents of the polymer compound of both oils, the technique of high performance size exclusion chromatography (HPSEC) was used. These compounds represent a set of constituents with diverse structures comprising polar and nonpolar lipid compounds (Figures 8 and 9).

Indeed, the content of DPTG varied between $1.2 \%$ and $0.2 \%$ before heating for both ROPO and ROPOE, respectively. During heating, the polymers rate underwent a significant increase as $4.3 \%, 10.7$, and $14.39 \%$ for unenriched (ROPO) after 7.5, 15, and $20 \mathrm{~h}$, respectively, while the lowest values of $2.7 \%, 6.9$, and $10.45 \%$, respectively, were detected by the supplementation of refined olive pomace oil with phenolic extract.

According to the obtained results (Table 2), the TPC and DPTG compounds are in compliance and are similar to previous results. ${ }^{18,19}$ Moreover, the enhancement of the oxidative resistance of refined oils was significantly observed with the merge of the olive leaf extract. This can be expounded by the effect of the phenolic compounds presented (oleuropein/ligstroside) in this extract, which act as natural antioxidants and have the ability to retard the oxidation of the oil.

Changes in the Specific Extinction Coefficients during Heating. What is worthwhile to mention is that the oxygen and the elevated temperature play a significant role in oil deterioration during frying. It is under room temperature that autoxidation happens by free radical mechanisms in which hydroperoxides play a major role. ${ }^{21}$

The ultraviolet spectrophotometric expressed as specific extinction coefficients was analyzed, suggesting the refined oils oxidation status. While $K_{232}$ correlates with the formation of conjugated dienes of polyunsaturated fatty acids, the $K_{270}$ values indicate very well the existence of primary and secondary oxidation products, namely conjugated trienes and carbonyl compounds.

Monitoring the evolution of specific extinction coefficients $\left(K_{232}\right.$ and $\left.K_{270}\right)$ as well the variation of specific extinction $\Delta K$ of the enriched and unenriched samples of refined heated olive-pomace oils was displayed in Table 3. 
Indeed, a considerable increase in $K_{232}$ and $K_{270}$ was detected for the unenriched refined oil samples. In this context, $K_{232}$ increased strongly as 5.84, 9.15; and 11.56 after 2.5, 10 , and $15 \mathrm{~h}$ of heating, respectively. This means that the content of primary oxidation products increases during frying. Similar behavior was observed for $K_{270}$ which rose sharply as $1.59,2.67$, and 2.73 , respectively.

Thus, refined olive-pomace oils are rich in secondary oxidation products and their contents increase at the end of the heating.

In order to confirm the obtained results, the measurement of the variation of extinction $\Delta K$ was carried out. This parameter draws a parallel with the oxidation state by identifying specific compounds oxidized at wavelengths of 266, 270, and $274 \mathrm{~nm}$.

Indeed, an increase in $\Delta K$ values for the unenriched samples is observed during $20 \mathrm{~h}$ of heating ranging from 0.97 to 3.02 (Table 3), showing that large quantities of conjugated dienes and trienes were formed during the heating process, which can also form polymers. These results accord well with those published previously. ${ }^{5,22}$

Nevertheless, the refined oils' oxidative resistance was considerably enhanced by the incorporation of phenolic compounds during heating. The enrichment of refined oils with the extract of olive leaf significantly decreases the values of the specific extinction coefficients at 232 and $270 \mathrm{~nm}$ compared to the unenriched oils.

In fact, the primary and secondary oxidation products decreased sharply from 15.38 to 10.01 and from 3.12 to 2.36 , respectively, after $20 \mathrm{~h}$ of heating. Furthermore, the specific extinction variation $\Delta K$ showed a low rate for the enriched oil as 2.29 compared to the unenriched one as 3.02 (Table 3).

In short, olive leaf extract has shown its ability to significantly reduce the formation of conjugated dienes and trienes in enriched refined oils over those formed in unenriched refined oils. It has an antioxidant activity that has improved the oxidative resistance of refined olive-pomace oils.

In conclusion, the enhancement of refined olive pomace oil with the extract of olive leaf exhibited a substantial decline in the formation of total polar compounds and the polymers, which are the best quality indicators of frying oil degradation.

Moreover, a remarkable resistance and a notable rise in the oxidative stability during 20 $\mathrm{h}$ of heating may be explicated by the abundance of the extract in oleuropein and ligstroside which are endowed with a powerful antioxidant effect. 
In fact, olive leaves, a byproduct, are considered as a potential source of high added values compounds. They are deemed as a prospective antioxidant source of natural origin. Their enrichment in refined oil industry may not only preserve their stability during frying and even storage, but also may remarkably reduce the formation of the potentially harmful products that contain the negative effects on consumers' health.

\section{AUTHOR INFORMATION}

Corresponding Author

Mohamed Bouaziz - Laboratoire d‘Électrochimie et Environnement, École Nationale d'Inge nieurs de Sfax, Université de Sfax, 3038 Sfax, Tunisia; Institut Supé rieur de Biotechnologie de Sfax, Universite de Sfax, 3038 Sfax, Tunisia; orcid.org/0000-0001-9107-7027;

Email: mohamed.bouaziz@,fsg.rnu.tn

\section{Authors}

Ibtissem Ben Hammouda - Laboratoire d‘Électrochimie et Environnement, École Nationale d'In'genieurs de Sfax, Université de Sfax, 3038 Sfax, Tunisia

Gloria Márquez-Ruiz - Instituto de Ciencia y Tecnología de Alimentos y Nutrición Consejo Superior de Investigaciones Científicas (ICTAN-CSIC), Madrid, Spain

Francisca Holgado - Instituto de Ciencia y Tecnología de Alimentos y Nutrición, Consejo Superior de Investigaciones Científicas (ICTAN-CSIC), Madrid, Spain

Ammar Sonda - Laboratoire d'E' lectrochimie et Environnement, E'cole Nationale d'Inge' nieurs de Sfax, Universite' de Sfax, 3038 Sfax, Tunisia

Krystyna Skalicka-Wozniak - Department of Pharmacognosy with Medicinal Plant Unit, Medical University in Lublin, 20- 093 Lublin, Poland

\section{Notes}

The authors declare no competing financial interest.

\section{ACKNOWLEDGMENTS}

The authors are grateful to the "Ministère de l'Enseignement Supérieur et de la Recherche Scientifique" (Laboratory LR14ES08), Tunisia, for its financial support for 
this research work. They would also extend their thanks to Mrs Leila Mahfoudhi, Teacher of English in the Faculty of Sciences of Sfax, for editing and polishing the language of the manuscript.

\section{ABBREVIATIONS}

BPCs, base peaks chromatograms; DAD, diode-array detection; DBE, double bond equivalents; DPPH, 2,2-diphenyl- 1picrylhydrazyl; DPTG, Dimers and polymers of triglycerides; HPLC, high-performance liquid chromatography; QTOF, quadrupole time-of-flight; MS, mass spectrometry; MS/MS, tandem mass spectrometry; ROPO, refined olive-pomace oil; RP, reversed-phase; TPC, Total polar compounds; UV, ultraviolet

\section{REFERENCES}

(1) Ben Hammouda, I.; Freitas, F.; Ammar, S.; Da Silva, M. D. R. G.; Bouaziz, M. Comparison and Characterization of Volatile Compounds as Markers of Oils Stability during Frying by HS-SPME-GC/ MS and Chemometric Analysis. J. Chromatogr. B: Anal. Technol. Biomed. Life Sci. 2017, 1068, 322-334.

(2) Ben Hammouda, I.; Zribi, A.; Ben Mansour, A.; Matthaus, B.; Bouaziz, M. Effect of Deep-Frying on 3-MCPD Esters and Glycidyl Esters Contents and Quality Control of Refined Olive Pomace Oil Blended with Refined Palm Oil. Eur. Food Res. Technol. 2017, 243 (7), 1219-1227.

(3) Ben Hammouda, I.; Triki, M.; Mattha“us, B.; Bouaziz, M. A Comparative Study on Formation of Polar Components, Fatty Acids and Sterols during Frying of Refined Olive Pomace Oil Pure and Its Blend Coconut Oil. J. Agric. Food Chem. 2018, 66 (13), 3514-3523.

(4) Ben Hammouda, I.; Marquez-Ruiz, G.; Holgado, F.; Freitas, F.; Da Silva, M. D. R. G.; Bouaziz, M. Comparative Study of Polymers and Total Polar Compounds as Indicators of Refined Oil Degradation during Frying. Eur. Food Res. Technol. 2019, 245 (5), 967-976.

(5) Zribi, A.; Gargouri, B.; Jabeur, H.; Rebaï, A.; Abdelhedi, R.; Bouaziz, M. Enrichment of Pan-frying Refined Oils with Olive Leaf Phenolic-rich Extract to Extend the Usage Life. Eur. J. Lipid Sci. Technol. 2013, 115 (12), 1443-1453. 
(6) Chen, X.; Zhang, Y.; Zu, Y.; Yang, L.; Lu, Qi.; Wang, W. Antioxidant Effects of Rosemary Extracts on Sunflower Oil Compared with Synthetic Antioxidants. Int. J. Food Sci. Technol. 2014, 49 (2), 385-391.

(7) Herrero, M.; Temirzoda, T. N.; Segura-Carretero, A.; Quirantes, R.; Plaza, M.; Ibanez, E. New Possibilities for the Valorization of Olive Oil By-Products. J. Chromatogr. A 2011, 1218 (42), 7511-7520.

(8) Bouaziz, M.; Grayer, R. J.; Simmonds, M. S. J.; Damak, M.; Sayadi, S. Identification and Antioxidant Potential of Flavonoids and Low Molecular Weight Phenols in Olive Cultivar Chemlali Growing in Tunisia. J. Agric. Food Chem. 2005, 53 (2), 236-241.

(9) Ahmad Farooqi, A.; Fayyaz, S.; Silva, A. S.; Sureda, A.; Nabavi, S. F.; Mocan, A.; Nabavi, S. M.; Bishayee, A. Oleuropein and Cancer Chemoprevention: The Link Is Hot. Molecules 2017, 22 (5), 705.

(10) Abidi, J.; Ammar, S.; Ben Brahim, S.; Skalicka-Wozniak, K.; Ghrabi-Gammar, Z.; Bouaziz, M. Use of Ultra-High-Performance Liquid Chromatography Coupled with Quadrupole-Time-of-Flight Mass Spectrometry System as Valuable Tool for an Untargeted Metabolomic Profiling of Rumex Tunetanus Flowers and Stems and Contribution to the Antioxidant Activity. J. Pharm. Biomed. Anal. 2019, 162, 66-81.

(11) Gargouri, B.; Ammar, S.; Zribi, A.; Mansour, A. B.; Bouaziz, M. Effect of Growing Region on Quality Characteristics and Phenolic Compounds of Chemlali Extra-Virgin Olive Oils. Acta Physiol. Plant. 2013, 35 (9), 2801-2812.

(12) Marquez-Ruiz, G.; Dobarganes, M. High-Performance Size-Exclusion Chromatography for Lipid Analysis in Organic Media. Lipid Anal. lipidomics new Technol. Appl. 2006, 205-238.

(13) Dobarganes, M. C.; Velasco, J.; Dieffenbacher, A. Determination of Polar Compounds, Polymerized and Oxidized Triacylglycerols, and Diacylglycerols in Oils and Fats: Results of Collaborative Studies and the Standardized Method (Technical Report). Pure Appl. Chem. 2000, 72 (8), 1563-1575.

(14) IOOC. International Olive Oil Council. Trade Standard Applying to Olive Oils and Olive Pomace Oils. Int. Olive Oil Counc. Madrid, Spain, 2003.

(15) Bouaziz, M.; Lassoued, S.; Bouallagui, Z.; Smaoui, S.; Gargoubi, A.; Dhouib, A.; Sayadi, S. Synthesis and Recovery of High Bioactive Phenolics from Table-Olive Brine Process Wastewater. Bioorg. Med. Chem. 2008, 16 (20), 9238-9246. 
(16) Ammar, S.; Contreras, M.; Gargouri, B.; Segura-Carretero, A.; Bouaziz, M. RPHPLC-DAD-ESI-QTOF-MS Based Metabolic Profiling of the Potential Olea Europaea by-Product "Wood" and Its Comparison with Leaf Counterpart. Phytochem. Anal. 2017, 28 (3), 217-229.

(17) Talhaoui, N.; Gomez-Caravaca, A. M.; Leon, L.; De la Rosa, R.; Segura-Carretero, A.; Fernandez-Gutierrez, A. Determination of Phenolic Compounds of 'Sikitita'Olive Leaves by HPLC-DADTOF- MS. Comparison with Its Parents 'Arbequina'and 'Picual'Olive Leaves. LWT-Food Sci. Technol. 2014, 58 (1), 28-34.

(18) Visioli, F.; Petroni, A.; Galli, C. Phenolic Compounds Extracted from Olive Oil Prevent Oxidation of Low Density Lipoproteins, and Inhibit Platelet Function and Platelet and Leukocyte Eicosanoid Production in Vitro. Oxidative Process. antioxidants 1994, 199-206.

(19) Cardoso, S. M.; Falcao, S. I.; Peres, A. M.; Domingues, M. R. Oleuropein/Ligstroside Isomers and Their Derivatives in Portuguese Olive Mill Wastewaters. Food Chem. 2011, 129 (2), 291-296.

(20) Aladedunye, F.; Przybylski, R. Degradation and Nutritional Quality Changes of Oil during Frying. J. Am. Oil Chem. Soc. 2009, 86 (2), 149-156.

(21) Dobarganes, M. C.; Velasco, J. Analysis of Lipid Hydroperoxides. Eur. J. Lipid Sci. Technol. 2002, 104 (7), 420-428.

(22) Marinova, E. M.; Seizova, K. A.; Totseva, I. R.; Panayotova, S. S.; Marekov, I. N.; Momchilova, S. M. Oxidative Changes in Some Vegetable Oils during Heating at Frying Temperature. Bulg. Chem. Commun. 2012, 44 (1), 57-63. 
Table 1. Phenolic Compounds Characterized Using the Negative Ionization Mode in Olive Leaves

\begin{tabular}{|c|c|c|c|c|c|c|c|c|}
\hline no & $\begin{array}{l}\mathrm{RT} \\
(\mathrm{min})\end{array}$ & $M(g / L)$ & $\begin{array}{l}\text { Exp. } m / z \\
\text { for }[\mathrm{M}-\mathrm{H}]^{-}\end{array}$ & formula & DBE & $\begin{array}{l}\text { UV } \\
(\mathrm{nm})\end{array}$ & main fragments via MS/MS & assignments \\
\hline 1 & 27.03 & 540.1843 & 539.1771 & $\mathrm{C}_{25} \mathrm{H}_{32} \mathrm{O}_{13}$ & 10 & $\begin{array}{l}242 \\
282\end{array}$ & $\begin{array}{l}\text { 403.1236;377.1241;371.0979;345.0981;327.0880;307.0822; } \\
275.0920 ; 223.0610 ; 179.0703 ; 165.0560 ; 149.0245 ; 139.0401\end{array}$ & oleuropein isomer a \\
\hline 2 & 28.17 & 540.1843 & 539.1774 & $\mathrm{C}_{25} \mathrm{H}_{32} \mathrm{O}_{13}$ & 10 & $\begin{array}{l}242 \\
282\end{array}$ & $\begin{array}{c}403.1262 ; 377.1263 ; 371.1002 ; 327.0915 ; 307.0842 ; 275.0578 \\
223.0629 ; 179.0703 ; 165.0573 ; 149.0260 ; 139.0412\end{array}$ & oleuropein isomer $b$ \\
\hline 3 & 29.45 & 524.1894 & 523.1823 & $\mathrm{C}_{25} \mathrm{H}_{32} \mathrm{O}_{12}$ & 10 & $\begin{array}{l}242 \\
282\end{array}$ & $\begin{array}{c}361.1291 ; 329.0899 ; 291.0877 ; 259.0977 ; 223.0610 ; 139.0374 \\
127.0401 ; 101.0244\end{array}$ & ligstroside \\
\hline
\end{tabular}


Table 2. Evolution of Total Polar Compounds and Polymers of Refined Olive-Pomace Oil Samples during Rancimat Heating ${ }^{a}$

\begin{tabular}{|c|c|c|c|c|}
\hline \multirow[b]{2}{*}{$\begin{array}{l}\text { frying } \\
\text { method }\end{array}$} & \multicolumn{4}{|c|}{ rancimat } \\
\hline & ТРС (\%) & DPTG (\%) & ТPC (\%) & DPTG (\%) \\
\hline time (h) & ROPO & ROPO & ROPOE & ROPOE \\
\hline 0 & 8 & 1.2 & 8 & 0.2 \\
\hline 2.5 & 9 & 1.9 & 8 & 1.1 \\
\hline 5 & 11.5 & 3.5 & 8.3 & 1.6 \\
\hline 7.5 & 12.8 & 4.3 & 10 & 2.7 \\
\hline 10 & 16.5 & 6.2 & 11.5 & 3.5 \\
\hline 12.5 & 18.5 & 6.9 & 15.5 & 5.8 \\
\hline 15 & 23.5 & 10.7 & 17 & 6.9 \\
\hline 20 & 26 & 14.39 & 24.75 & 10.45 \\
\hline
\end{tabular}

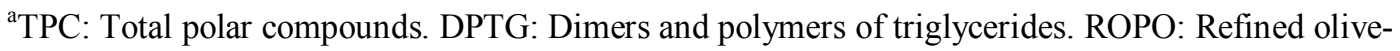
pomace oil. ROPOE: Refined olive-pomace oil enriched with phenolic extract. 
Table 3. Conjugated Diene Values $\left(K_{232}\right.$ and $\left.K_{270}\right)$ and the Variation of Extinction $\Delta K$ of Refined Olive-Pomace Oil with and without the Addition of Olive Leaf Extract during Heating with Rancimat

\begin{tabular}{ccccccc} 
& \multicolumn{9}{c}{ rancimat } \\
\cline { 2 - 7 } time (h) & \multicolumn{2}{c}{$\mathrm{K}_{270}$} & \multicolumn{2}{c}{$\mathrm{K}_{232}$} & \multicolumn{2}{c}{$\Delta K$} \\
2.5 & 1.59 & 0.79 & 5.84 & 4.37 & 0.97 & 0.73 \\
5 & 2.12 & 1.37 & 7.16 & 5.55 & 1.92 & 1.28 \\
7.5 & 2.51 & 1.9 & 9.01 & 5.92 & 2.32 & 1.79 \\
10 & 2.67 & 2.04 & 9.15 & 6.48 & 2.60 & 1.90 \\
12 & 2.71 & 2.06 & 9.91 & 8.60 & 2.75 & 2.04 \\
15 & 2.73 & 2.10 & 11.56 & 9.44 & 2.77 & 2.15 \\
20 & 3.12 & 2.36 & 15.38 & 10.01 & 3.02 & 2.29 \\
\hline
\end{tabular}




\section{FIGURE CAPTIONS}

Figure 1. (A) Base peak chromatogram (BPC) of the ethanolic extract of olive leaves obtained by RP-UHPLC-DAD-QTOF-MS, (B) ESI-BPC-MS spectrum, and (C) UV spectrum of the different isomers of the $[\mathrm{M}-\mathrm{H}]^{-}$ion at $(\mathrm{m} / \mathrm{z} 539.1771)$.

Figure 2. (A) MS spectrum and (B) spectrum $\mathrm{MS}^{2}$ highlighting the main fragments of the isomer (a) of the $[\mathrm{M}-\mathrm{H}]^{-}$ion at $(\mathrm{m} / \mathrm{z} 539.1771)$.

Figure 3. Proposed scheme for fragmentation of the $[\mathrm{M}-\mathrm{H}]^{-}$ion at $(\mathrm{m} / \mathrm{z} 539.1771)$.

Figure 4. ESI-BPC-MS spectrum of the $[\mathrm{M}-\mathrm{H}]^{-}$ion at $(\mathrm{m} / \mathrm{z} 523.1823)$.

Figure 5. (A) MS spectrum and (B) $\mathrm{MS}^{2}$ spectrum highlighting the main fragments of the $[\mathrm{M}-\mathrm{H}]^{-}$ion at $(\mathrm{m} / \mathrm{z} 523.1823)$.

Figure 6. Proposed scheme for fragmentation of the $[\mathrm{M}-\mathrm{H}]^{-}$ion at $(\mathrm{m} / \mathrm{z}$ 523.1823).

Figure 7. Antiradical activity of the extract of the olive leaves.

Figure 8. Distribution of polar compounds in refined olive-pomace oil unenriched with phenolic extract after $20 \mathrm{~h}$ of heating based on HPSEC. TGO, triglycerides oligomers; TDM, triglycerides dimers; TG, triglycerides; DG, diglycerides.

Figure 9. Distribution of polar compounds in refined olive-pomace oil enriched with phenolic extract after $20 \mathrm{~h}$ of heating based on HPSEC. TGO, triglycerides oligomers; TDM, triglycerides dimers; TG, triglycerides; DG, diglycerides. 
Figure 1.
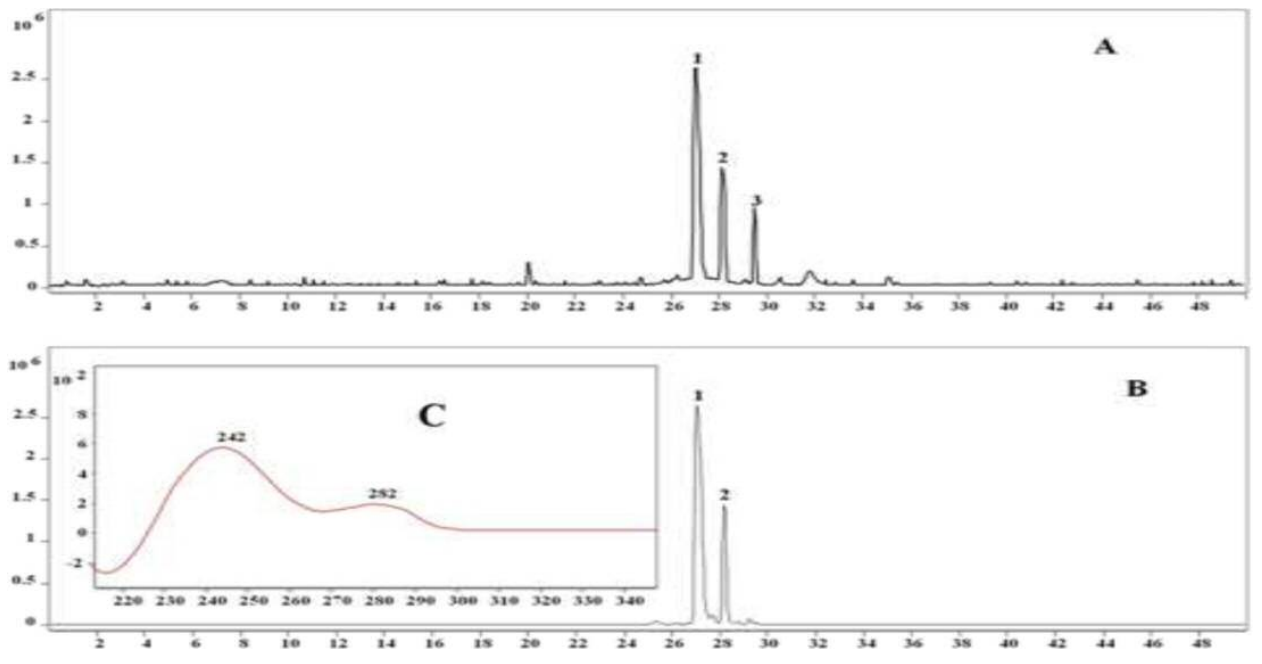
Figure 2.
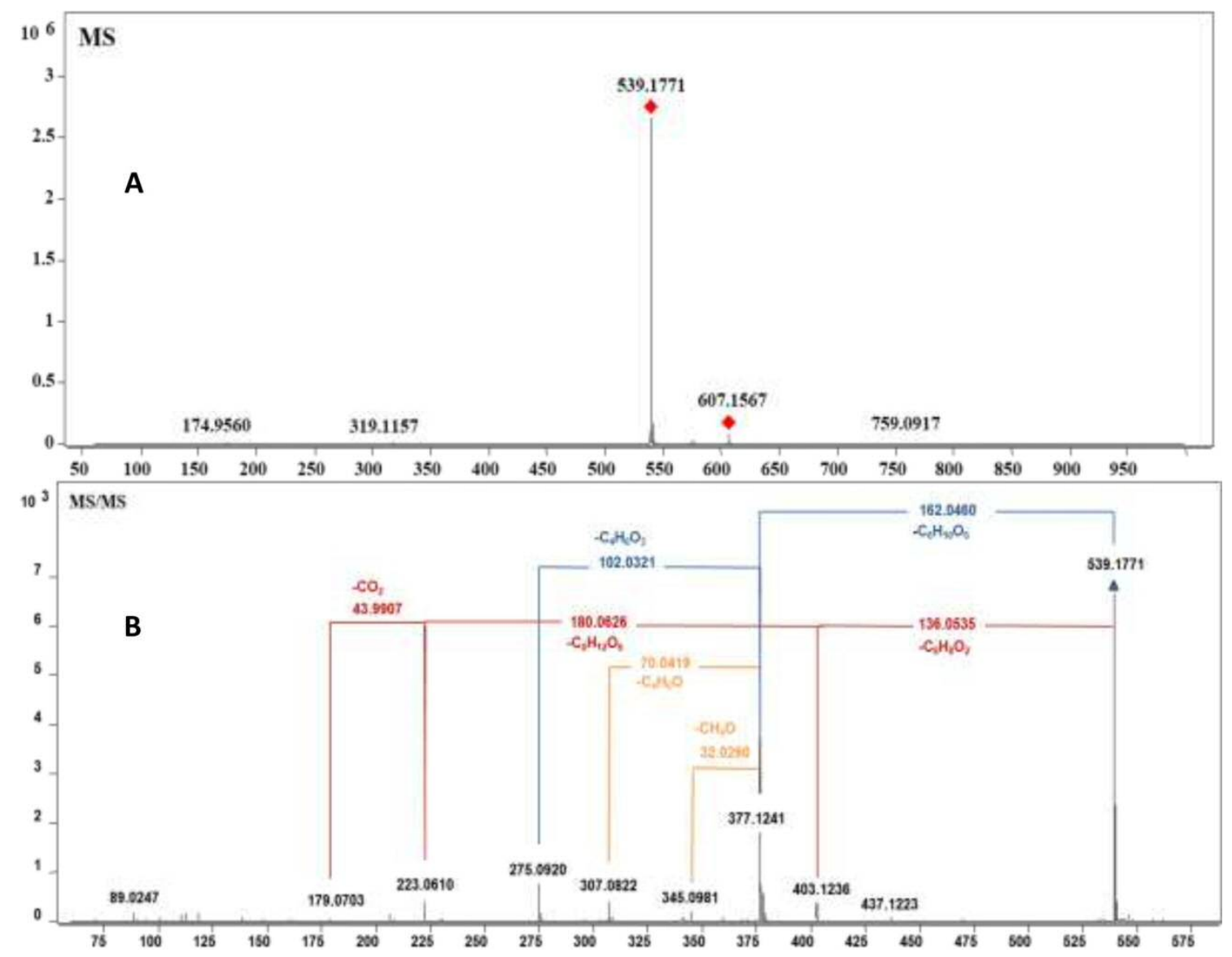

https://dx.doi.org/10.1021/acs.jafc.0c05509

J. Agric. Food Chem. 2020, 68, 12039-12047 


\section{Figure 3.}

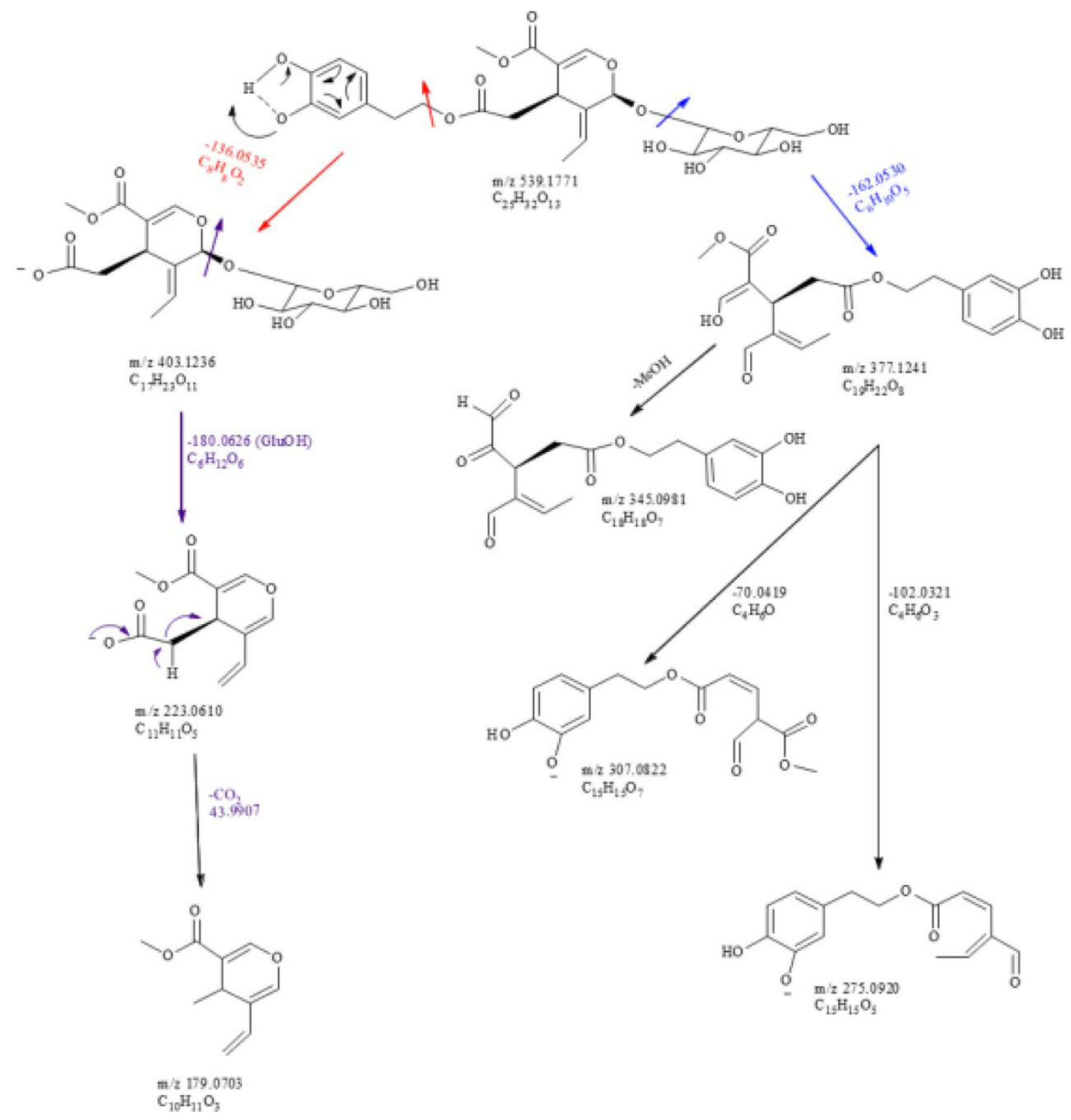




\section{Figure 4.}

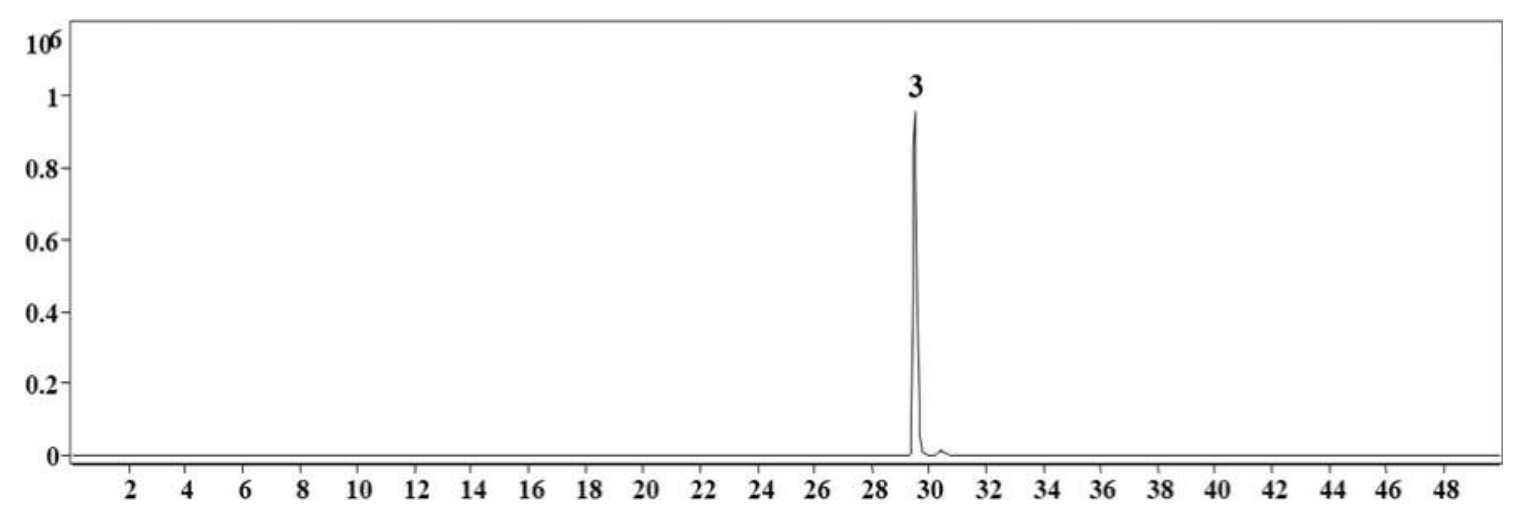


Figure 5.
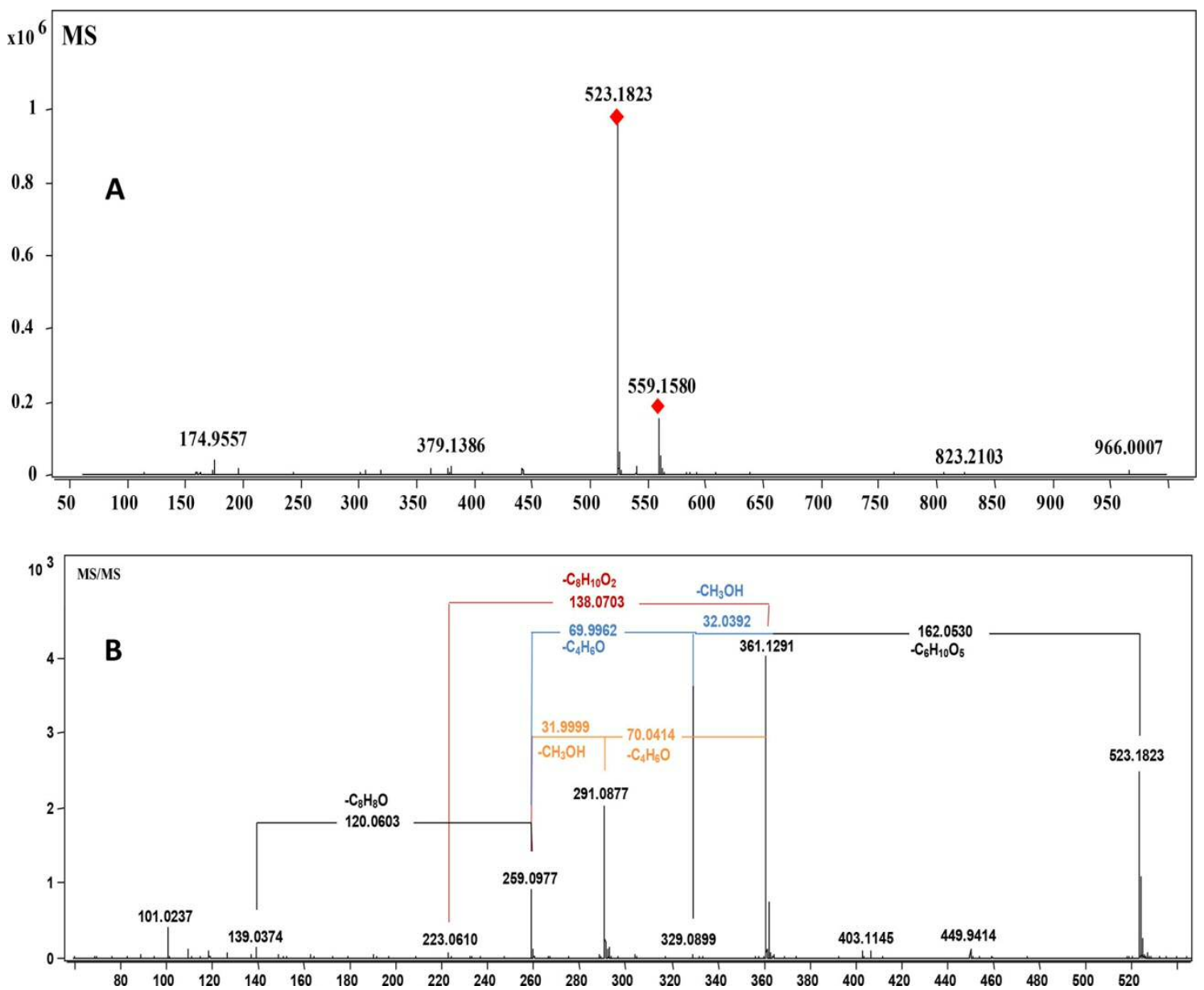

https://dx.doi.org/10.1021/acs.jafc.0c05509

J. Agric. Food Chem. 2020, 68, 12039-12047 


\section{Figure 6.}

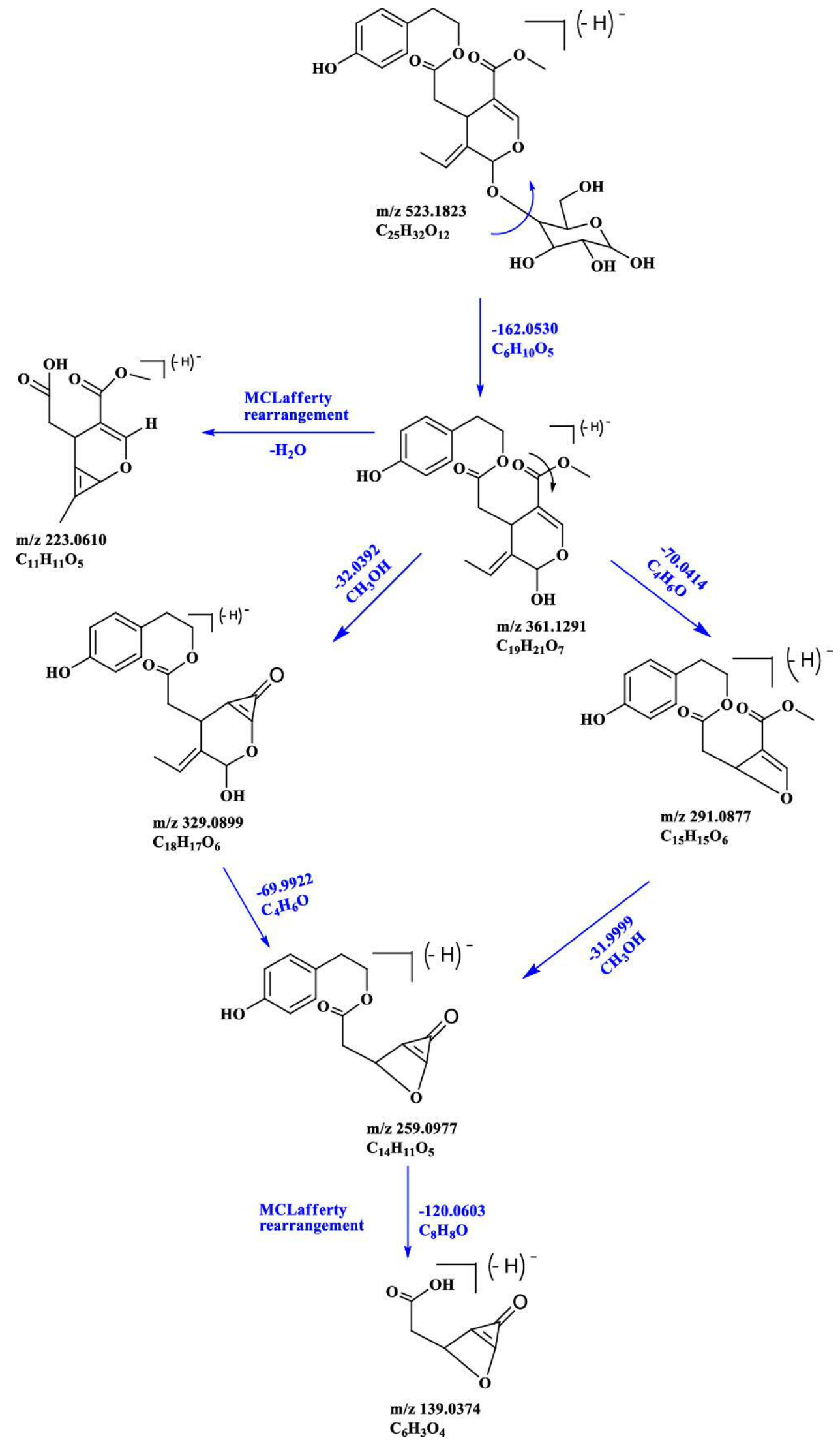

https://dx.doi.org/10.1021/acs.jafc.0c05509

J. Agric. Food Chem. 2020, 68, 12039-12047 
Figure 7.

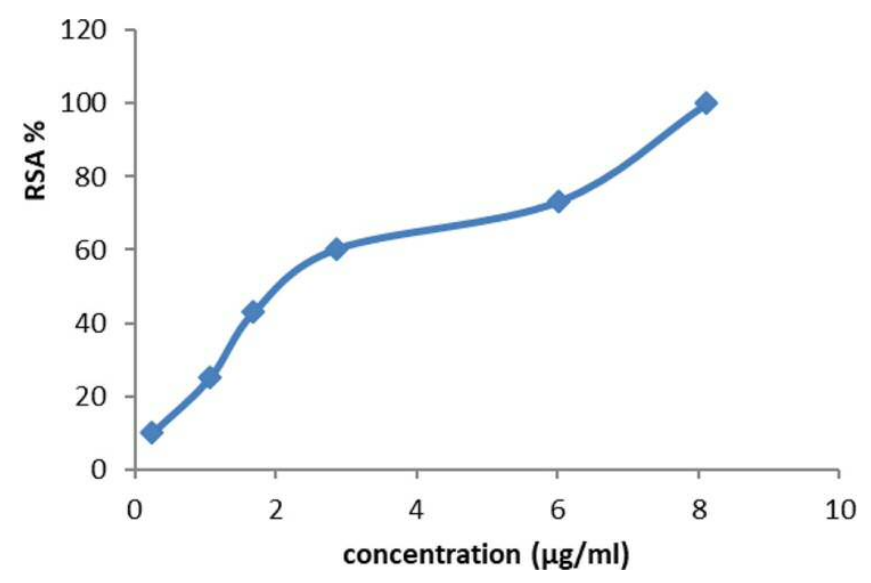

https://dx.doi.org/10.1021/acs.jafc.0c05509

J. Agric. Food Chem. 2020, 68, 12039-12047 
Figure 8.

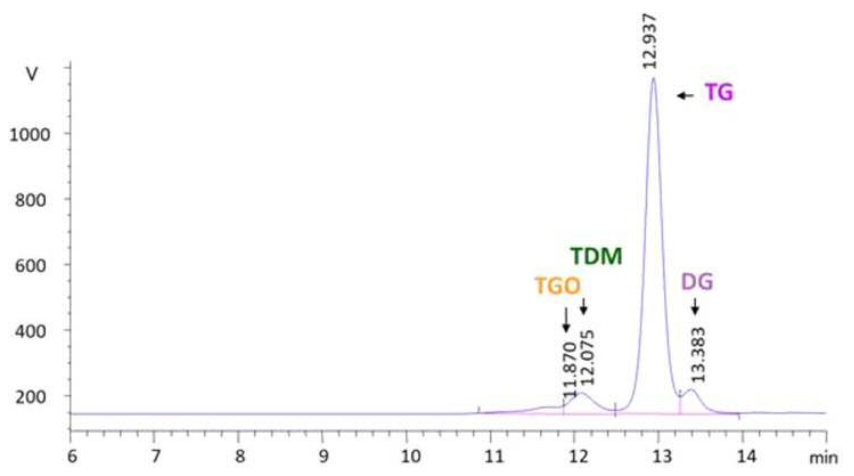

https://dx.doi.org/10.1021/acs.jafc.0c05509

J. Agric. Food Chem. 2020, 68, 12039-12047 
Figure 9.

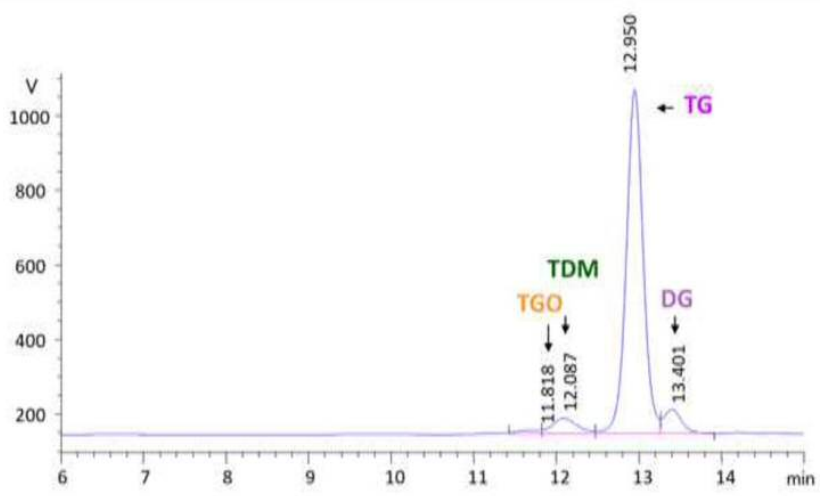

https://dx.doi.org/10.1021/acs.jafc.0c05509

J. Agric. Food Chem. 2020, 68, 12039-12047 